A. Ceschin ${ }^{1}$, L. Angelilli ${ }^{1}$, F.E. Hernández Pérez ${ }^{1}$, I. Boxx ${ }^{2}$, H.G. $\operatorname{Im}^{1}$

Study on coherent structures for high turbulence burner, AIAA Scitech 2021 Forum, (AIAA 2019-1733).

${ }^{1}$ King Abdullah University of Science and Technology (KAUST), CCRC, Thuwal 23955-6900, Saudi Arabia

${ }^{2}$ Institute for Combustion Technology, German Aerospace Center (DLR), Stuttgart 70569, Germany

The AIAA version of the paper is accessible at https://doi.org/10.2514/6.2021-1849

On the AIAA web page

https://arc.aiaa.org/doi/pdf/10.2514/6.2021-1849

the interested reader can find other material published by AIAA 


\title{
Study on coherent structures for high turbulence burner
}

\author{
Alberto Ceschin*1 ${ }^{* 1}$ Lorenzo Angelilli ${ }^{\dagger 1}$, Francisco E. Hernández Pérez ${ }^{\ddagger 1}$, Isaac Boxx ${ }^{\S 2}$, and Hong G. Im ${ }^{\llbracket 1}$ \\ ${ }^{1}$ King Abdullah University of Science and Technology (KAUST), CCRC, Thuwal 23955-6900, Saudi Arabia \\ ${ }^{2}$ German Aerospace Center (DLR), Stuttgart 70569, Germany
}

\begin{abstract}
A numerical study on the coherent structure generation and analysis by means of $Q$ and $\lambda_{2}$ criteria is conducted. The case under study is represented by the HiPilot burner, experimentally investigated at the University of Michigan for the classification of turbulent premixed flames based on Karlovitz number. Large eddy simulations of a cold jet are performed to gain insights into the dynamics and characteristics of the high Reynolds number turbulent flow, especially in the section that is optically inaccessible for measurements.
\end{abstract}

\section{Introduction}

Research on turbulent premixed flames is a fundamental step for the design and the development of low-emissions and efficient combustion devices [1]. Ideally, a few condensed parameters should characterize the combustion regime due to the flame/turbulence interaction in order to apply assumptions that can lead to simplified mathematical models. Historically, these parameters have been identified as the ratio between velocity fluctuations and the corresponding unstretched laminar flame speed, as well as the ratio of integral length scale to the laminar flame thickness. Besides, non-dimensional numbers such as the Reynolds (Re), Karlovitz (Ka) and Damköhler (Da) numbers are typically used for this purpose [2]. The Borghi diagram [3] was designed to classify the turbulent combustion regimes according to the Karlovitz number, which is the ratio of chemical time scale to Kolmogorov time scale. Three turbulent regimes are identified in the diagram, separated by values of Ka equal to 1 and 100. They are in order: corrugated flamelets, thin reaction zone and distributed combustion regimes [4]. According to the Klimov-Williams criterion [5], the value of $\mathrm{Ka}=100$ divides the last two mentioned regimes, setting the theoretical achievement of the distributed regime. However, this value is still under investigation in the turbulent combustion community [6].

For such scope, the Michigan HiPilot burner was designed [7], intending to study the turbulent flame structures generated by a methane-air mixture at high values of Reynolds and Karlovitz numbers. Simultaneously acquired planar laser-induced fluorescence (PLIF) images [8] of $\mathrm{CH}$ radicals, formaldehyde $\left(\mathrm{CH}_{2} \mathrm{O}\right)$ and hydroxyl $(\mathrm{OH})$ were used to identify and measure the preheat and reaction layer thickness. Reported experimental results suggest that the previous broadened preheat, yet thin reaction layer, can extend for Ka up to five times higher than the Klimov-Williams criterion, extending the application of the flamelet model. Moreover, from recent publications reported in the review by Driscoll et al. [9], it appears that, contrary to what has been previously theorized, broadening does not begin when the Kolmogorov eddy size equals the laminar flame thickness. Further investigation, supporting experiments with simulation, is required to carefully address these new hypothesis.

The above results instigate further research on this topic. In particular, computational studies on these experiments are needed to gain deeper insights into the characteristics of the high Reynolds number flow. More specifically, to determine whether the presence of coherent structures can partially justify the high values of velocity fluctuations $\left(u^{\prime}\right)$ and integral length scale $\left(L_{x}\right)$ reported with turbulent Reynolds number $\left(R e_{T}\right)$ up to 99000 and turbulent Karlovitz number $\left(K a_{T}\right)$ up to 533. To this end, the presence and role of coherent structures is studied by compressible large eddy simulation (LES) in the present work. The choice of this approach is due to its capability to represent the larger three-dimensional unsteady turbulent motions and ability to capture flow separation and vortex shedding without fully resolving the small scales and, hence, considerably reducing the computational effort.

The next section presents the mathematical and numerical model, followed by the description of the configuration and test case. Subsequently, the results are discussed.

\footnotetext{
*PhD Student, corresponding author, Clean Combustion Research Center, alberto.ceschin@ kaust.edu.sa, AIAA Student member.

$\dagger \mathrm{PhD}$ Student, Clean Combustion Research Center, lorenzo.angelilli@kaust.edu.sa, AIAA Student member.

${ }^{\ddagger}$ Research scientist, Clean Combustion Research Center, francisco.hernandezperez.1@kaust.edu.sa, AIAA Member.

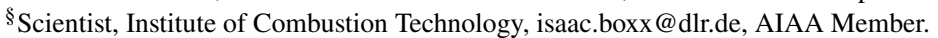

IIProfessor, Clean Combustion Research Center, hong.im@kaust.edu.sa, AIAA Associate fellow.
} 


\begin{tabular}{ccccccc}
\hline Flow rate & Plate & Jets & $U_{0}[\mathrm{~m} / \mathrm{s}]$ & $u^{\prime}[\mathrm{m} / \mathrm{s}]$ & $L_{x}[\mathrm{~mm}]$ & $\mathrm{Re}_{T}$ \\
\hline 3 & B & off & 17 & 5.9 & 7.9 & 3000 \\
3 & B & on & 21 & 7.1 & 9.2 & 4200 \\
\hline
\end{tabular}

Table 1 Parameters characterizing the cases considered in the study.

\section{Numerical model}

The large eddy simulation (LES) equations for the filtered quantities are the following[10]:

$$
\begin{gathered}
\frac{\partial \bar{\rho}}{\partial t}+\frac{\partial\left(\bar{\rho} \tilde{u}_{j}\right)}{\partial x_{j}}=0 \\
\frac{\partial\left(\bar{\rho} \widetilde{u}_{i}\right)}{\partial t}+\frac{\partial\left(\bar{\rho} \widetilde{u}_{i} \widetilde{u}_{j}\right)}{\partial x_{j}}=-\frac{\partial \bar{p}}{\partial x_{i}}+\frac{\partial}{\partial x_{j}}\left(2 \bar{\mu} \widetilde{S}_{i j}\right)+\frac{\partial L_{i j}}{\partial x_{j}} \\
\frac{\partial \bar{\rho} \tilde{e}}{\partial t}+\frac{\partial\left(\bar{\rho} \tilde{e} \tilde{u}_{j}\right)}{\partial x_{j}}=\frac{\partial}{\partial x}\left(\tilde{u}_{j} \bar{p}+\tilde{q}_{j}-\tilde{u}_{i} \tilde{\tau}_{i j}^{t o t}\right),
\end{gathered}
$$

and the stress tensor is defined as

$$
\widetilde{S}_{i j}=\frac{1}{2}\left(\frac{\partial \widetilde{u}_{i}}{\partial x_{j}}+\frac{\partial \widetilde{u}_{j}}{\partial x_{i}}\right)-\frac{1}{3} \delta_{i j} \frac{\partial \widetilde{u}_{k}}{\partial x_{k}} .
$$

Equations 1, 2. and 3 represent the conservation of mass, momentum, and energy. Furthermore, the ${ }^{-}$symbol denotes filtered quantities, while the ${ }^{\sim}$ symbol denotes density-weighted (Favre) filtered quantities. In the above equations, $\rho$ is the density, $u_{j}$ is the velocity component in the direction $j, p$ is the pressure, $e$ is the total energy, $q$ is the heat flux, $\tau_{i j}^{t o t}$ is the total stress tensor, and $\delta_{i j}$ is the Dirac delta.

The subgrid scale stress tensor, $L_{i j}$, must be modeled. According to the eddy viscosity assumption, it is expressed as

$$
L_{i j}=\widetilde{u_{i}} \widetilde{u_{j}}-\widetilde{u_{i} u_{j}} \sim 2 v_{t} \widetilde{S}_{i j},
$$

where $v_{t}$ is the turbulent viscosity, which also requires a model. For this work, the wall-adapting local eddy-viscosity (WALE) [11] model is adopted. This model is based on the construction of the tensor invariant $\mathcal{S}_{i j}^{d}$ and models the turbulent viscosity as

$$
v_{t}=\left(C_{w} \Delta\right)^{2} \frac{\left(\mathcal{S}_{i j}^{d} \mathcal{S}_{i j}^{d}\right)^{3 / 2}}{\left(\widetilde{S}_{i j} \widetilde{S}_{i j}\right)^{5 / 2}+\left(\mathcal{S}_{i j}^{d} \mathcal{S}_{i j}^{d}\right)^{5 / 4}},
$$

where $C_{w}$ is a constant obtained by assuming that the model gives the same ensemble-average subgrid kinetic energy dissipation as the classical Smagorinsky model.

\section{Configuration and test case description}

The burner, object of study for this work, is represented by the HiPilot: a piloted, Bunsen-type burner developed to reach extreme levels of turbulence [8, 12]. Figure 1 shows the burner configuration, and in the following a brief description of the role of each section is provided.

The main flow enters the burner after a glass bed pipe with a diameter of $93 \mathrm{~mm}$. This pipe creates a quasi-flat profile that is broken by an interchangeable blockage plate that has been found to produce rather large turbulence intensity. Alternatives for turbulence generation are represented by either grid or moving plates, but velocity fluctuations hardly exceed values above $10 \%$ of of the mean velocity. In this case, the claimed percentage is up to $30 \%$ [12]. Although different plates for blockage ratio have been adopted throughout the history of the burner, the work will focus on the plate "B", with a blockage ratio of 0.85 . Marshall reported the mean azimuthal velocity to increase monotonically with the blockage ratio and advised not to exceed blockage ratio greater than 0.93 because of suspected -but not identified- large turbulent structures. The dynamics of these claimed structured has not be disclosed yet and the aim of this manuscript is to accomplish this goal.

Then, a convergent-divergent duct with throat diameter of $12 \mathrm{~mm}$ stretches the vortices generated after the blockage plate in the axial direction. At the throat of the convergent-divergent duct, six radial jets with a diameter of $1.27 \mathrm{~mm}$ 


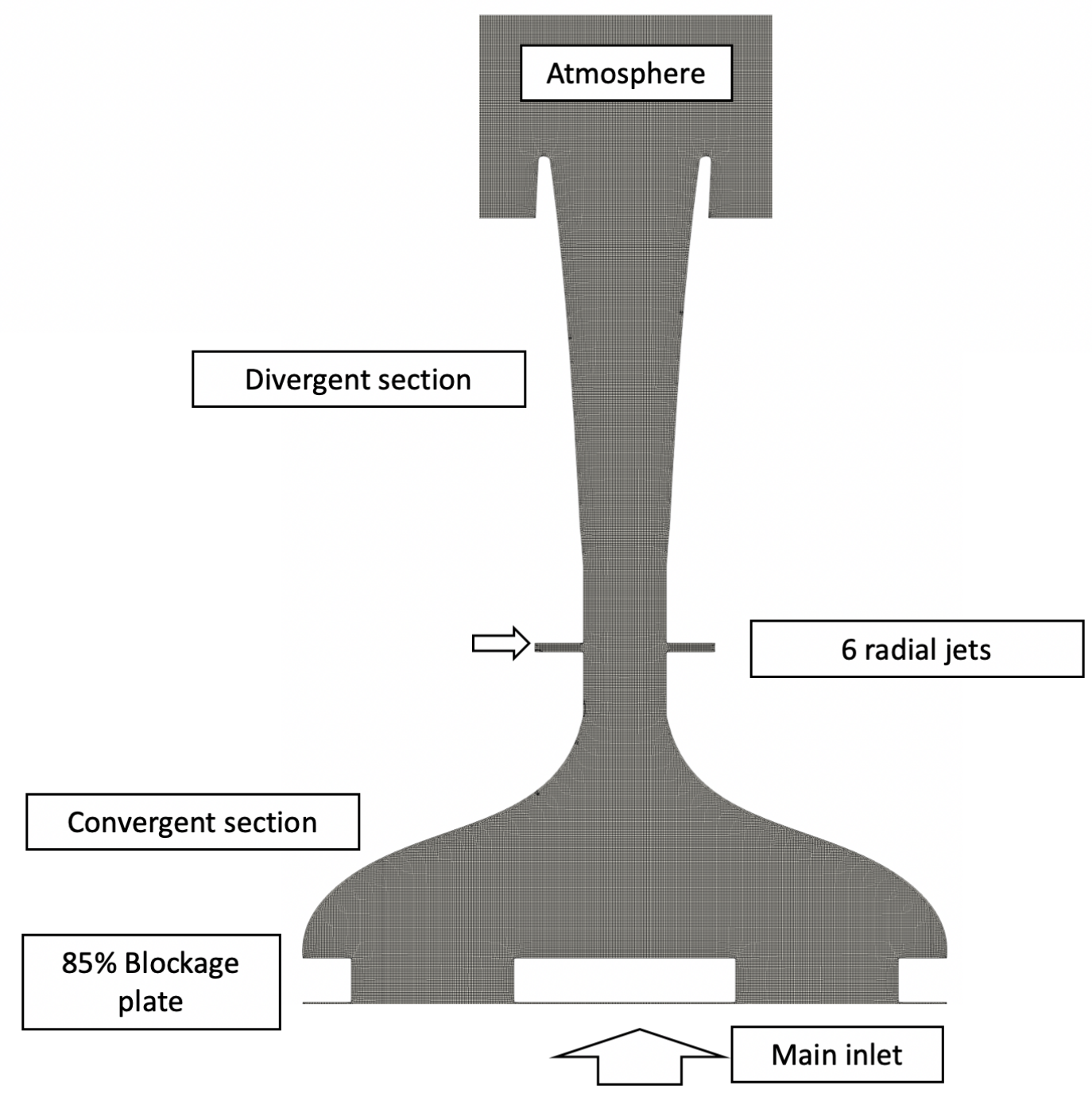

Fig. 1 Details of the longitudinal section of the HiPilot burner mesh.

are present to intentionally break the flow pattern and create additional turbulence. The exit of the divergent duct, of diameter equal to $21.6 \mathrm{~mm}$, releases the flow to the atmosphere, surrounding the jet with a co-flow. The influence of the co-flow is left to future work, but there is the hypothesis that it could be entrained by large eddies at the nozzle outlet, modifying the composition of the mixture. This question has been addressed in [13]. The radial jets account for the 6\% of the total mass flow rate. More details regarding the burner configuration can be found in [8].

This work focuses on two significant cases which have been experimentally investigated. Table 1 lists the experimental velocimetry results collected by Skiba $e t$ al. [8], where $U_{0}$ is the center line velocity at $5 \mathrm{~mm}$ above the nozzle, $u^{\prime}$ is the velocity fluctuation level, $L_{x}$ is the longitudinal length scale, and $R e_{T}$ is the turbulent Reynolds number. The aim of this simulation campaign is to reproduce the high turbulence level reported in the aforementioned references and perform the coherent structure analysis by means of $Q$ and $\lambda_{2}$ criteria described in the next section. The influence of the radial jets on the flow pattern is studied in the two variants of case 3B.

This complex geometry has been created with Salome [14], and subsequently meshed in CFMesh [15], a powerful meshing tool based on the OpenFOAM [16] framework and suitable for irregular geometries. The mesh employs up to 35 million cells to reach a resolution of $200 \mu \mathrm{m}$ in all the burner geometry and sampling outlet, with a total of 5 refinement levels. Mesh skewness does not exceed 2 and non-orthogonality 55, with an average below 2. Nevertheless, 
one non-orthogonal corrector is applied as a safe approach. The equations are implemented in rhoPimpleFoam, a transient solver for compressible turbulent flow available in the open-source OpenFOAM framework. The choice of a compressible solver has been motivated by a Mach number of 0.4 in the throat in case $3 \mathrm{~B}$. Three outer and two inner correctors are applied to couple the solution variables in the PIMPLE loop. Second order discretizations in space and time were utilized, and the time step is conditioned by a Courant number lower than 0.3. Such a low value is recommended in a large eddy simulation. The sampling region extends for 2 diameters after the nozzle exit, consequently domain has been extended to 10 nozzle diameters downstream to reduce the influence of boundary conditions.

\section{Results}

Since a direct comparison with experimental data has not been possible due to the lack of public consistent measurements, radial profiles of $\overline{u_{z}}$ and related fluctuations $u_{z}^{\prime}$ at different distances from the nozzle exit are qualitatively compared to the previous experimental results [13] of a similar configuration.

Figure $2 \mathrm{a}$ shows the radial profiles of $\overline{u_{z}}$. Two observation can be made: (i) the profile obtained at $5 \mathrm{~mm}$ from the nozzle exit presents a weak asymmetry, coherently with the experimental data and (ii) fluctuations reach more than one third of the mean velocity because of the peculiar geometry of the burner. Since the characteristic time scale of the fluid dynamics is large due to the swirl effect induced by the burner design, the averages may require a rather long time to reach convergence. However, since the far field has a satisfactory profile, the simulation can be considered to be sufficiently evolved for analysis.

The other component to characterize the $\operatorname{Re}_{T}$ together with the velocity fluctuations is the integral length scale. The computed values are generally smaller than the ones reported (7.9 and $9.2 \mathrm{~mm}$ ), but have the same order of magnitude and an axial component bigger than the radial one, stressing the anisotropy of the large scale motions. To understand the reason behind these high values, existence of core vortex and large turbulent structures has been hypothesized. Hence, two validated criteria for vortex core identification have been used: the $Q$-criterion developed by Hunt et al. in [17] and the $\lambda_{2}$ criterion [18]. The $Q$-criterion is based on the second invariant of the velocity gradient $\nabla u$ :

$$
Q=\frac{1}{2}\left(\|\Omega\|^{2}-\|S\|^{2}\right),
$$

where $\|\Omega\|^{2}=\left[\operatorname{tr}\left(\Omega \Omega^{T}\right)^{1 / 2}\right],\|S\|^{2}=\left[\operatorname{tr}\left(S S^{T}\right)^{1 / 2}\right]$ and

$$
\Omega_{i j}=\frac{1}{2}\left(\frac{\partial u_{i}}{\partial x_{j}}-\frac{\partial u_{j}}{\partial x_{i}}\right)
$$

is the vorticity tensor. Hunt et al. [17] locate an eddy along an isocontour with a defined $Q>0$ condition. This method is not able to capture properly the structure close to the wall, for this reason the $\lambda_{2}$ is also considered. The $\lambda_{2}$ criterion [18] is based on the acceleration tensor $D_{i j}$, that can be decomposed into a symmetric and a skew-symmetric part:

$$
D_{i j}=\underbrace{\left[\frac{D S_{i j}}{D t}+S_{i k} S_{k j}+\Omega_{i k} \Omega_{k j}\right]}_{\text {symmetric }}+\underbrace{\left[\frac{D \Omega_{i j}}{D t}+S_{i k} \Omega_{k j}+\Omega_{i k} S_{k i}\right]}_{\text {skew-symmetric }} .
$$

Since

$$
D_{i j}=-\frac{1}{\rho} p \delta_{i j}+v S_{i j},
$$

taking only the symmetric part of the tensor, the transport equation for $S_{i j}$ is obtained:

$$
\frac{D S_{i j}}{D t}+S_{i k} S_{k j}+\Omega_{i k} \Omega_{k j}-v S_{i j}=-\frac{1}{\rho} p \delta_{i j} .
$$

Moreover, since the vortices are located where a local minimum of the pressure is achieved, it is sufficient that only the second eigenvalue of $S_{i k} S_{k j}+\Omega_{i k} \Omega_{k j}$ be negative.

As reported by Dubief [19], both criteria have been found to produce similar results, even though the $Q$-criterion sometimes produces cleaner structures with less noise in the background. Hence, this one is used for illustrating the flow pattern and coherent vortical structures. Note that the pressure contour was attempted too, but the resulting images are not as illustrative. 
Figure 3 shows the $Q$-criterion corresponding to a representative snapshot of case 3B. The highlighted part represents the contour surface where $Q=3 \times 10^{8}$, which has been chosen to represent main vortex cores without an excessive level of noise as compared to smaller positive values of $Q$.

The evolution of the vortices generated by the blockage plate is now described step-by-step along the five different stages (Figs. 3 and 5 .

1) The choice of the aforementioned value of $Q$ reveals how vortex tubes are formed because of the blockage plate. Similarly to the classic fluid dynamics problem of the backward facing step [19], vortices are generated at the leading edge due to the shear layer, but when the detachment occurs, the coherent structures slide towards the central axis of the geometry. In the backward facing example, detached vortices become parallel to the main direction of the flow after 5-to-7 characteristic length scales. In contrast, for this case, eddies already follow the orientation of the driving flow and are transported without breaking. This is the peculiarity of the HiPilot burner.

2) Since the flow is parallel to the leading edge, the configuration allows the eddies to grow and slide towards the central axis. Figure 2 shows the axial mean velocity taken from a section normal to the central axis after half centimeter, which discloses the recirculation zones.

3) These continuous long vortex tubes are further stretched in the convergent section, unveiling the reason of large integral scales $L_{x}$ at the nozzle exit. For cases with a higher flow rate [20], the length scale can reach values up to 37 and $41 \mathrm{~mm}$, almost twice the nozzle diameter. Furthermore, it is noticeable that vortex tube generation happens in only one of the two leading edges of the passage in an axisymmetric way. Sharing the same orientation but at different locations, these vortex structures combine, feeding a central counter-current vortex due to continuity, and rise through the convergence section without breaking. An example of the path is reported in the Figure with a red line. At this stage, the presence of Taylor instabilities might be deduced by the presence of counter-rotating dynamics.

4) Radial jets, accounting for $6 \%$ of the total mass injected with the $75 \%$ of the main flow velocity, do not have enough momentum to penetrate the flow field and break consistently the vortex tubes (Fig. 57. Nevertheless, they compress the vortices along the centerline causing collisions.

5) About 2 throat diameters above the radial jet, the presence of the aforementioned Taylor instabilities and the energy dissipation due to the compression from the lateral jets are responsible at this point to break the long vortex tube into smaller coherent structures of the same length scale measured close to the nozzle exit.
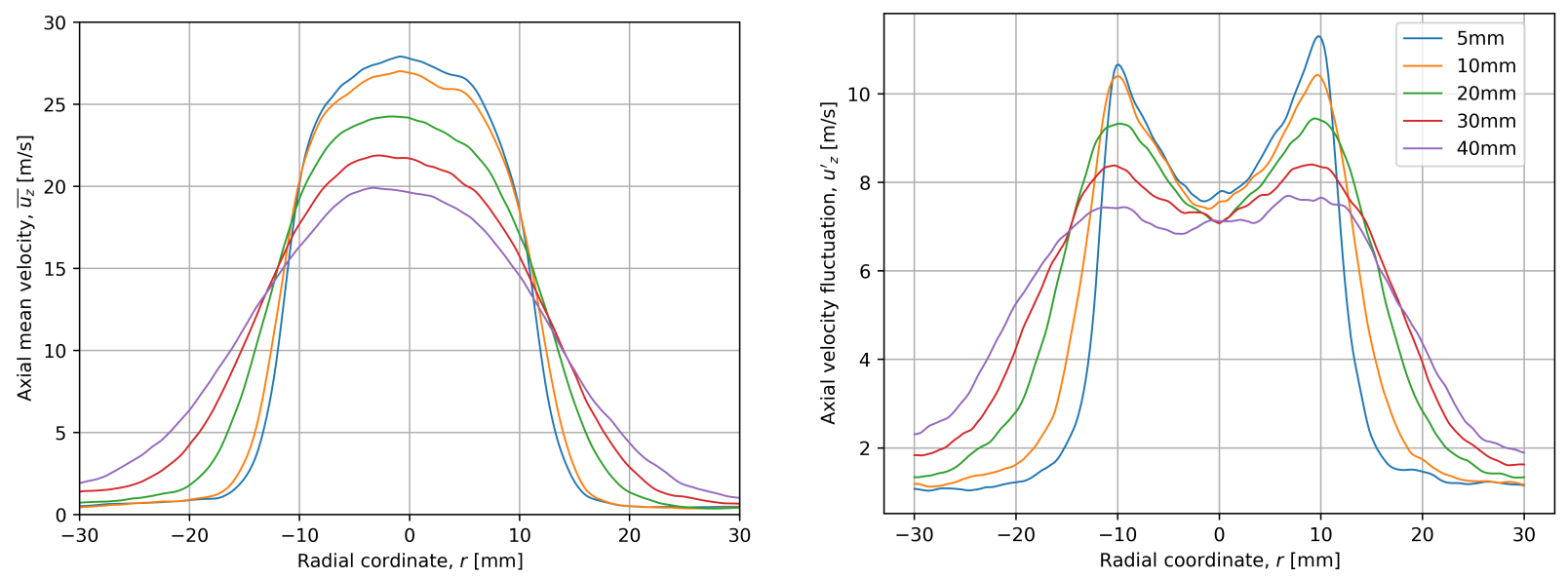

Fig. 2 Case 3B: Axial velocity mean and fluctuation along the radial coordinate. Profiles at 5, 10, 20, 30 and $40 \mathrm{~mm}$ from the nozzle exit. The nozzle diameter is $21.6 \mathrm{~mm}$.

\section{Conclusion}

The formation of large structures in the burner geometry has been investigated. It has been shown that they could be responsible of large turbulence fluctuation at the outlet nozzle. Nevertheless, a more rigorous and quantitative study by means of modal decomposition will be addressed in the future with the data produced, as soon as experimental validation results will be available. Large structures definitively play a role in turbulence characterization and their 


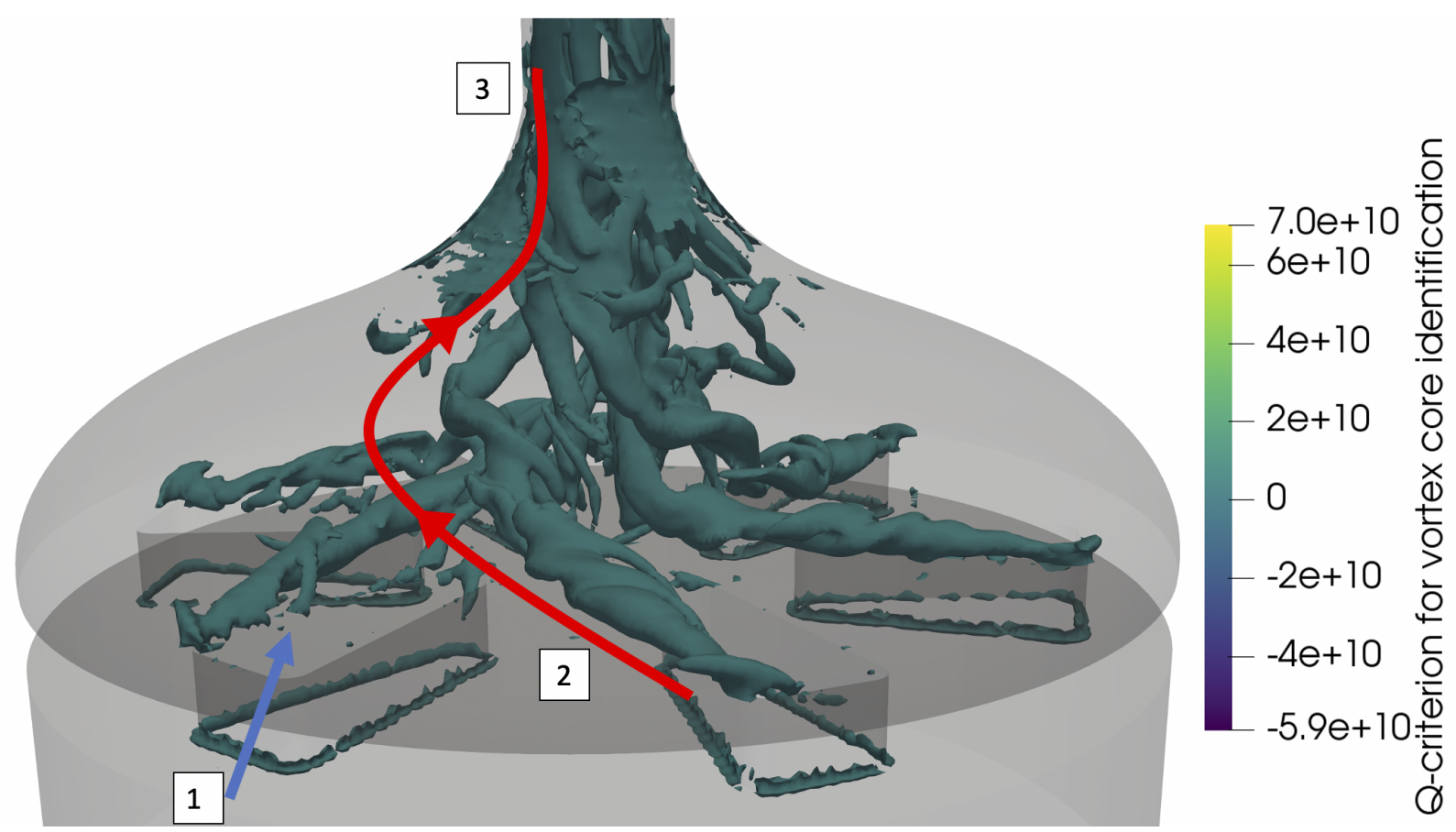

Fig. 3 Instantaneous snapshot of $Q$-criterion isocontour at $Q=3 \times 10^{8}$, case $3 \mathrm{~B}$. Leading edge is indicated by blue line (1), vortex tube path (2) by red line, up the end of the convergent section (3).

interaction with the flame has to be further investigated.

\section{Acknowledgments}

The authors acknowledge the support of King Abdullah University of Science and Technology (KAUST). Computational resources were provided by the KAUST Supercomputing Laboratory (KSL). This project has received funding from the European Research Council (ERC) under the European Union's Horizon 2020 research and innovation programme (Grant agreement No. 682383).

\section{References}

[1] Veynante, D., and Vervisch, L., "Turbulent combustion modeling," Progress in Energy and Combustion Science, Vol. 28, No. 3, 2002, pp. 193 - 266. https://doi.org/https://doi.org/10.1016/S0360-1285(01)00017-X URL http://www.sciencedirect.com/ science/article/pii/S036012850100017X

[2] Peters, N., “Turbulent combustion,”, 2001.

[3] Borghi, R., On the Structure and Morphology of Turbulent Premixed Flames, chapter and pages, pp. 117-138. https: //doi.org/10.1007/978-1-4684-4298-4_7 URL https://doi.org/10.1007/978-1-4684-4298-4_7

[4] Peters, N., "Laminar flamelet concepts in turbulent combustion," Symposium (International) on Combustion, Vol. 21, No. 1, 1988, pp. 1231 - 1250. https://doi.org/https://doi.org/10.1016/S0082-0784(88)80355-2. URL http://www.sciencedirect.com/ science/article/pii/S0082078488803552 twenty-First Symposuim (International on Combustion).

[5] Williams, F., "Criteria for existence of wrinkled laminar flame structure of turbulent premixed flames," COMBUST. AND FLAME; U.S.A.; DA. 1976; VOL. 26; NO 2; PP. 269-270; BIBL. 4 REF., 1976.

[6] Im, H. G., Arias, P. G., Chaudhuri, S., and Uranakara, H. A., "Direct Numerical Simulations of Statistically Stationary Turbulent Premixed Flames," Combustion Science and Technology, Vol. 188, No. 8, 2016, pp. 1182-1198. https://doi.org/10.1080/ 00102202.2016.1198789 URL https://doi.org/10.1080/00102202.2016.1198789 
[7] Temme, J., Wabel, T. M., Skiba, A. W., and Driscoll, J. F., "Measurements of premixed turbulent combustion regimes of high Reynolds number flames," 53rd AIAA Aerospace Sciences Meeting, 2015, p. 0168.

[8] Skiba, A. W., Wabel, T. M., Carter, C. D., Hammack, S. D., Temme, J. E., and Driscoll, J. F., "Premixed flames subjected to extreme levels of turbulence part I: Flame structure and a new measured regime diagram," Combustion and Flame, Vol. 189, 2018, pp. 407-432.

[9] Driscoll, J. F., Chen, J. H., Skiba, A. W., Carter, C. D., Hawkes, E. R., and Wang, H., "Premixed flames subjected to extreme turbulence: Some questions and recent answers," Progress in Energy and Combustion Science, Vol. 76, 2020 , p. 100802.

[10] Pope, S. B., “Turbulent flows,”, 2001.

[11] Nicoud, F., and Ducros, F., "Subgrid-scale stress modelling based on the square of the velocity gradient tensor," Flow, Turbulence and Combustion, Vol. 62, No. 3, 1999, pp. 183-200.

[12] Marshall, A., Venkateswaran, P., Noble, D., Seitzman, J., and Lieuwen, T., "Development and characterization of a variable turbulence generation system," Experiments in Fluids, Vol. 51, No. 3, 2011, p. 611.

[13] Wabel, T. M., Skiba, A. W., and Driscoll, J. F., "Evolution of turbulence through a broadened preheat zone in a premixed piloted Bunsen flame from conditionally-averaged velocity measurements," Combustion and Flame, Vol. 188, 2018, pp. 13-27.

[14] Code, A., "Salome-Meca,", 2018.

[15] Juretic, F., “cfMesh User Guide,” Creative Fields, Ltd, Vol. 1, 2015.

[16] Weller, H. G., Tabor, G., Jasak, H., and Fureby, C., "A tensorial approach to computational continuum mechanics using object-oriented techniques," Computers in physics, Vol. 12, No. 6, 1998, pp. 620-631.

[17] Hunt, J. C., Wray, A. A., and Moin, P., “Eddies, streams, and convergence zones in turbulent flows," 1988.

[18] Jeong, J., and Hussain, F., “On the identification of a vortex,” Journal of fluid mechanics, Vol. 285, 1995, pp. 69-94.

[19] Dubief, Y., and Delcayre, F., “On coherent-vortex identification in turbulence,” Journal of turbulence, Vol. 1, No. 1, 2000, pp. 011-011.

[20] Wabel, T. M., “An experimental investigation of premixed combustion in extreme turbulence," PhD Thesis, 2017. 


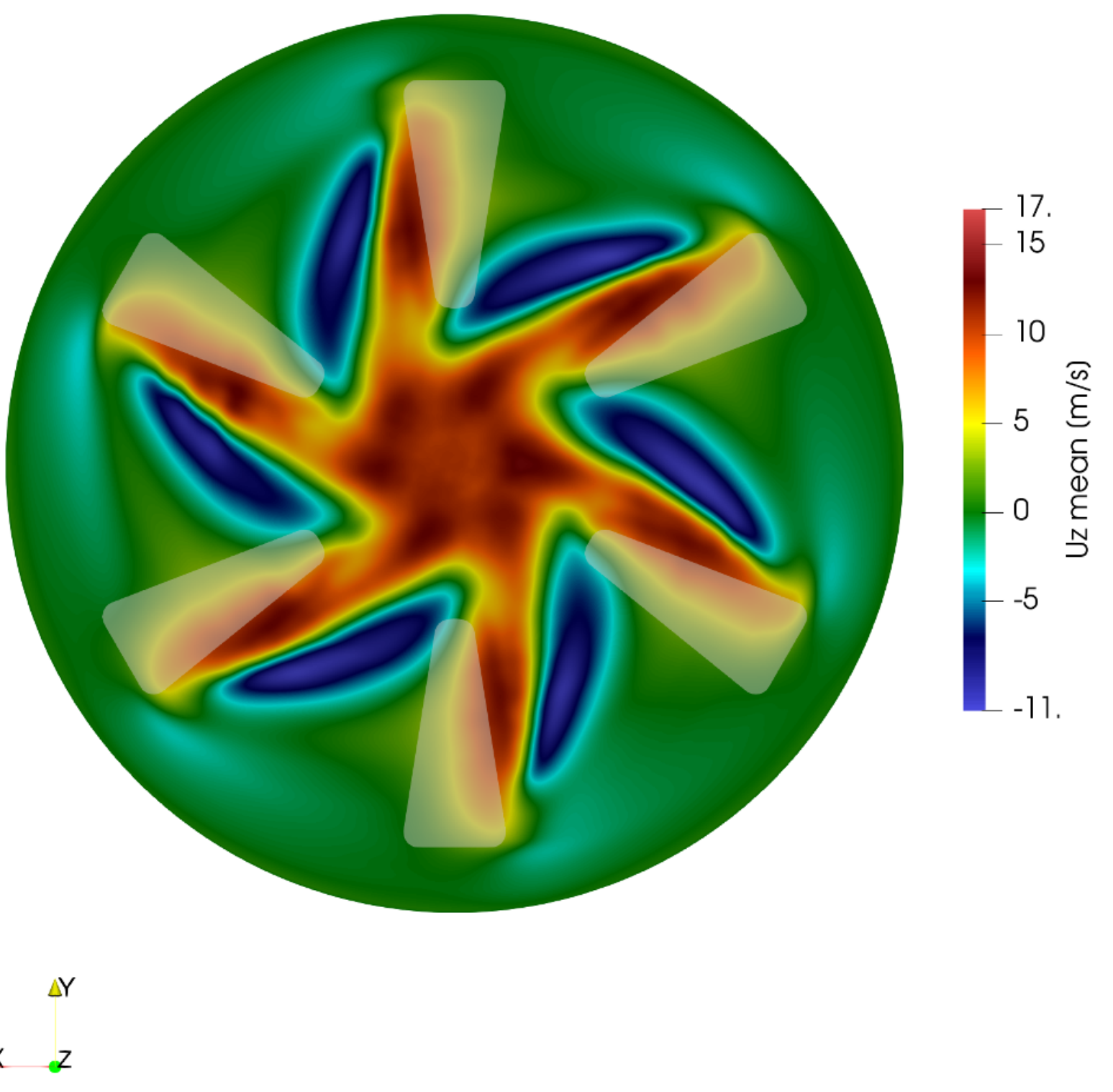

Fig. 4 Mean axial velocity $0.5 \mathrm{~cm}$ above the blockage plate (bottom view). Warm (red) areas represent the fluid exiting through the passage and going upward to the convergent section. Cold (blue) areas represent the recirculation zone. Flow passage in matte white. 


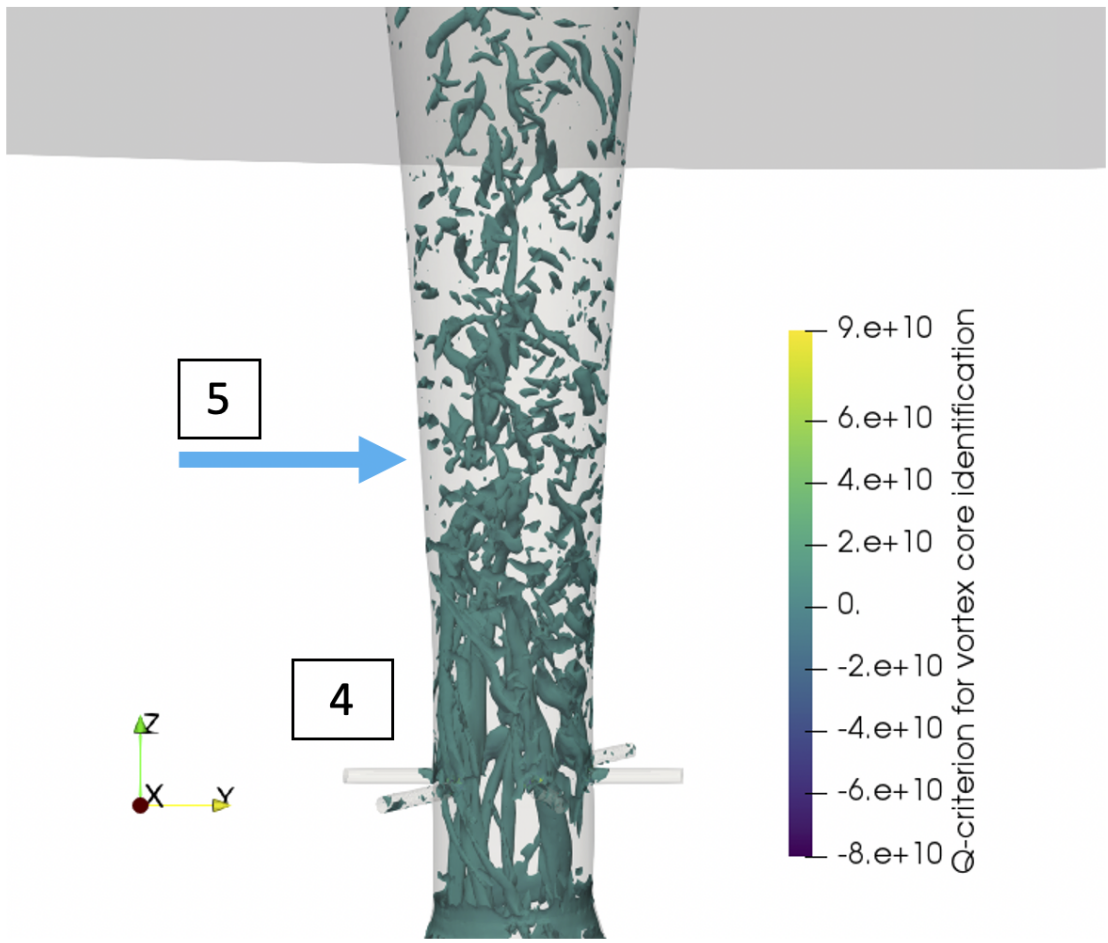

Fig. 5 Instantaneous snapshot of $Q$-criterion isocontour at $Q=3 \times 10^{8}$, case 3B. Radial jet locations (4) and vortex tube break-up (5) region are emphasized. 